\title{
Desafios terapêuticos das "bolsites" Bolsite na Visão de um Cirurgião
}

\section{Fabio Vieira Teixeira Rogerio Saad-Hossne}

Até meados dos anos 60, a proctocolectomia com ileostomia definitiva tipo Brooke era aceita como a técnica padrão no tratamento da retocolite ulcerativa inespecífica (RCUI). Apesar de $90 \%$ dos pacientes submetidos a operação se adaptarem ao procedimento, o fato de se manter uma ileostomia definitiva trazia prejuízos inegáveis à qualidade de vida dos pacientes operados.

Desde problemas clínicos como lesões cutâneas periestomais devido ao contato do efluente ileal com a pele, foram relatados problemas relacionados à absorção de água e eletrólitos (desidratação, cálculos renais) até alterações psicossociais tanto da esfera sexual (impotência em cerca de 5 a 10\%). quanto de alteração da imagem corporal ${ }^{1}$.

No final da década de 60, Nils Koch descreveu o uso de um reservatório intestinal feito com $45 \mathrm{~cm}$ de íleo terminal anastomosado a pele. Com isso ele criou uma ileostomia continente, ou seja, não ocorreria à drenagem do efluente ileal continuamente e o paciente poderia escolher a hora e lugar para esvaziar o reservatório (pouch) por meio de auto-cateterização.

Todavia, com o reservatório de Koch, complicações relacionadas ao procedimento começaram a ser descritos na literatura: dificuldade de esvaziamento do reservatório, incontinência do reservatório e necessidade de remoção do reservatório em 10\% a 40\% dos pacientes.

A confecção de um reservatório intestinal descrita pela primeira vez por Koch foi, sem dúvida, a operação precursora da a procto- 
colectomia com reservatório e anastomose anal. Além disso, pela primeira vez foi descrito a inflamação do reservatório de Koch conhecido por pouchitis ou bolsite, como veremos a diante, é a principal complicação pós-operatório de pacientes submetidos a cirurgia com reservatório ileal².

No final de década de 70 dois cirurgiões do St. Mark's Hospital de Londres, Alan Parks e John Nichols, descreveram a técnica da proctocolectomia com reservatório ileal e anastomose anal (ileal pouchanal anastomosis - IPAA) para o tratamento cirúrgico da RCUI. ${ }^{3}$ Por permitir a ressecção completa da doença, por restaurar o transito intestinal e manter um reservatório fecal semelhante ao reto, rapidamente, em pouco tempo, a IPAA se tornou o procedimento de escolha no tratamento cirúrgico da $\mathrm{RCUI}^{3}$.

A proctocoletomia com reservatório ileal é a operação de escolha no tratamento cirúrgico da RCUI. É bem descrito na literatura médica que a mucosa ileal sofre várias alterações morfológicas após a ser transformada em reservatório de fezes. O contato constante das fezes com a mucosa ileal promove uma série de alterações inflamatórias o que leva a mudança da arquitetura histológica do epitélio: a mucosa de intestino delgado com longas vilosidades, se achata e se transforma em uma mucosa sem vilosidades e com criptas profundas, característico do cólon. A essa modificação epitelial se dá o nome de metaplasia colônica.

Na verdade, além da alteração morfológica, a mucosa do reservatório sofre também uma modificação na composição do muco. Tem sido descrito que ao invés de sialomucina, característico do epitélio de intestino delgado, a mucosa do pouch é composta de sulfomucina, muco encontrado no cólon ${ }^{4-5}$. Alguns pesquisadores acreditam que essa metaplasia colônica observada no reservatório seja fisiológica e não tenha nenhum tipo de representatividade clínica. Todavia, outros, apontam que a alteração na histologia intestinal, poderia também criar um ambiente propício para que a inflamação se estabelecessa novamente. Essa é a teoria da retocolite ulcerativa de novo na bolsa ileal.

A bolsite (pouchitis em Inglês) é a mais frequente complicação observada após uma IPAA. A prevalência cumulativa pode variar de $23 \%$ a $46 \%$, com um incidência anual de $40 \%{ }^{6}$.

De forma didática, o diagnóstico deve ser dividido em bolsite agu- 
da e bolsite crônica. A bolsite aguda usualmente é tratada por meio de antibióticos e aparece dias ou semanas após o fechamento da ileostomia de proteção. A inflamação seria, na verdade, em decorrência de uma modificação da microbiota e/ou no aumento microrganismos ${ }^{6}$.

A disbiose (alteração da composição das bactérias comensais) é um dos principais fatores na gênese da bolsite. Por outro lado, a presença de patógenos como fungos, vírus e bactéria como o Clostridium difficile, devam ser sempre considerados na causa da bolsite aguda. Outras espécies de bactérias podem estar relacionadas a infecção da bolsa: Incertae Sedis, Clostridial cluster e Lachnospiraceae ${ }^{6-9}$.

Portadores de bolsite aguda respondem satisfatoriamente a antibioticoterapia por 2 semanas, normalmente, a ciprofloxacina e o metronidazol são as drogas mais usadas ${ }^{6,9}$. Após o fechamento da ileostomia de proteção, pacientes que apresentem sintomas de diarreia, dor abdominal, febre e não melhorem após tratamento com antibiótico, o diagnóstico de complicações cirúrgicas relacionado a bolsa deve ser sempre considerado.

Como já dito nesse capítulo, a bolsa ileal favorece a estase fecal com consequente aumento da proliferação bacteriana expondo a mucosa intestinal a um novo microambiente e com isso ocasionado alterações morfológicas no epitélio conhecida por metaplasia colônica.

Parece que a metaplasia colônica está relacionada a disbiose, principalmente, por bactérias redutoras de sulfato. Já foi descrito alterações bioquímicas no muco (sulfomucina) de reservatórios intestinais semelhantes as observadas em portadores de retocolite ulcerativa. Uma vez que o muco é responsável pela proteção da mucosa, alterações nessa glicoproteína poderiam favorecer a quebra da barreira epitelial com consequente aumento de permeabilidade. ${ }^{6}$ 0 aumento da permeabilidade favoreceria o aumento da imunogenicidade e consequente desencadeamento do processo inflamatório.

Sob o ponto de vista dos resultados cirúrgicos, algumas considerações devem ser feitas e pontuadas.

Desde a sua concepção, a IPAA tem oferecido aos pacientes com RCUI, colite indeterminada, FAP e Doença de Crohn uma qualidade muito boa, associada a excelentes resultados funcionais.

A analise copilada dos principais estudos da literatura, evidencia 
uma taxa média de complicações agudas e tardias aceitáveis (10 a 25\%), assim como taxas as morbidades (22 a 33\%); a formação de fistulas também deve ser considerada, com taxas medias de 4 a $10 \%$. Neste sentido a experiência da equipe cirurgia é o fator determinante para os bons resultados do procedimento. A taxa de falha bolsa após IPAA ocorre entre $6 \%$ a $12 \%$ dos pacientes, sendo que a excisão da bolsa ileal é baixa variando de 1 a 3\% Quanto aos indicies médios de sucesso da bolsa observados a longo prazo, isto é, 5,10 , 15 e 20 anos é 95, 90, 87 e 85\%, sendo que a principal causa de falha da bolsa é a ocorrência de sepse pélvica (5 a 11\%), tendo como principais fatores de risco a imunossupressão com corticóides ${ }^{10-15}$.

Porém, apesar destes resultados globais aqui descritos, duas casuísticas mundiais destacam-se na cirurgia de IPAA; a da Clevaland Clinic e da Mayo Clinic, que juntas totalizam 5592 pacientes.

$\mathrm{Na}$ analise do grupo de Cleveland ${ }^{10}(\mathrm{n}=3707)$, maior casuística mundial, as taxas de complicações precoces foi de 33,5\% e 29,1\% de complicações tardias, apesar desta morbidade de quase $66 \%$, a maioria dos pacientes (96\%) apresentou altos indicies de qualidade de vida, semelhante àquelas relatadas em outros centros. A incidência de pouchitis foi de 33,8\% , sendo que 15,9\% evoluíram para bolsite crônica. As taxa de falha da bolsa após IPAA ocorreram em 6\% a 12\% dos pacientes após 10 e 15 anos, sendo que as causas principais foram causas foram sepse pélvica, fístula e Doença de Crohn. As taxas de incontinência também são baixas (2 a 8\%), porém com aumento das taxas de acordo com a idade e com o envelhecimento. Os indicies de qualidade de vida (saúde geral, os níveis de disposição, satisfação com a cirurgia e etc) e os resultados funcionais após 10 anos da cirurgia são altos (96,3\%). Os autores concluem que é uma excelente opção cirúrgica, com índice de qualidade de vida alto e com grande satisfação por parte dos pacientes coma cirurgia ${ }^{10}$.

Resultados semelhantes foram encontrados pelo grupos da Mayo Clinic, onde ao longo de 20 anos foram estudados 1885 pacientes $^{11}$. A principal complicação após IPAA encontrada neste grupo foi a bolsite, cujas taxas de incidência chegaram a 48\% após 10 anos e 70\% após 20 anos. A sepse pélvica foi a principal causa da falha bolsa em todos os pacientes; sua ocorrência pode ser demonstrada em 2 momentos distintos. 0 primeiro ocorreu no início do curso 
de pós-operatório, provavelmente secundário a uma complicação pós-operatória (fistulas). O segundo foi evidenciado apos anos de evolução e foi atribuído a doença de Crohn. Independentemente do momento as taxas foram baixas ( $<5 \%)$. Todos os pacientes deste estudo, segundo os autores, tem tido excelentes taxas de qualidade de vida após IPAA, saudáveis e com bons resultados em todos os escores de qualidade, confirmando os dados de outros estudos. Os autores concluem que os resultados clínicos e funcionais após IPAA são excelentes e estáveis a longo prazo (20 anos) e que esta cirurgia evoluiu ao longo do tempo para uma operação confiável e de escolha para colite ulcerativa crônica apesar das altas taxas de bolsite ${ }^{11}$.

Alguns detalhes técnicos são fundamentais para os bons resultados cirúrgicos, sendo que os mesmos podem interferir direta ou diretamente na fisiopatologia da bolsite. A anastomose da bolsa com a região anal/retal deve ser completamente livre de tensão, o fluxo sanguíneo deve ser muito bom para evitar a isquemia crônica, a checagem dos anéis da anastomose mecânica devem estar íntegros, e o teste de vazamento de ar (borracheiro) deve ser negativo.

Desta forma, sob o ponto de vista cirúrgico e na visão do cirurgião a bolsite é uma complicação esperada e muito frequente após a IPAA, apesar disso, este procedimento cirúrgico atinge os principais objetivos no tratamento da RCUI: erradicação da doença e proteção do paciente da evolução para uma doença maligna, com altas taxas de qualidade de vida e excelentes resultados funcionais a longo prazo.

\section{Referências bibliográficas}

1. Kelly KA, Dozois RR. Chronic ulcerative colitis - Cap 38. In: Mayo Gastrointestinal Surgery, Kelly KA, Saar MG, Hinder RA. $1^{\text {st }}$ ed. Elsevier Saunders, Philadelphia pp 533-552, 2003

2. Koch NG, Kewenter J. Intraabdominal reservoir in ileostomy. Nord Med. 1971 Jul 22;86(29):887-8.

3. Parks AG, Nicholls RJ. Proctocolectomy without ileostomy for ulcerative colitis. Br Med J. 1978 Jul 8;2(6130):85-8.

4. Garcia-Armengol J11, Hinojosa J, Lledo S, Roig JV, Garcia-Granero E, Martinez B.Prospective study of morphologic and functional changes with time in the mucosa of the ileoanal pouch:functional appraisal using transmucosal potential differences. Dis Colon Rectum. 1998 Jul;41(7):846-53.

5. Teixeira FV, Daud D, Eleuterio ML, Silva MD, Kelly KA. Morphology of ileal and jejunal pouches used as rectal substitutes. Dis Colon Rectum. 2003 Mar;46(3):373-6.

6. Shen B. Pouchitis: what every gastroenterologist needs to know. Clin Gastroenterol Hepatol. 2013 Dec;11(12):1538-49.

7. Komanduri S, Gillevet PM, Sikaroodi M, et al. Dysbiosis in pouchitis: evidence of unique microfloral patterns in pouch in- flammation. Clin Gastroenterol Hepatol 2007;5:352-360.

8. Lim M, Sagar P, Finan P, et al. Dysbiosis and pouchitis. Br J Surg 2006;93:1325-1334.

9. Holubar SD, Cima RR, Sandborn WJ, et al. Treatment and prevention of pouchitis after ile- 
al pouch-anal anastomosis for chronic ulcerative colitis. Cochrane Database Syst Rev 2010; 6:CD001176.

10. V. W. Fazio, R. P. Kiran, F. H. Remzi, et al., "Ileal pouch anal anastomosis: analysis of outcome and quality of life in 3707 patients," Annals of Surgery, vol. 257, no. 4, pp. 679-685, 2013.

11. D. Hahnloser, J. H. Pemberton, B. G. Wolff, D. R. Larson, B. S. Crownhart, and R. R. Dozois, "Results at up to 20 years after ileal pouch-anal anastomosis for chronic ulcerative colitis," British Journal of Surgery, vol. 94, no. 3, pp. 333-340, 2007.

12. D. S. Pardi and W. J. Sandborn, "Systematic review: the manage- ment of pouchitis," Alimentary Pharmacology \& Therapeutics, vol. 23, no. 8, pp. 1087-1096, 2006.

13. O. Zmora, M. Natanson, I. Dotan et al., "Long-term functional and quality-of-life outcomes after IPAA in children," Diseases of the Colon \& Rectum, vol. 56, no. 2, pp. 198-204, 2013.

14. A. P. Meagher, R. Farouk, R. R. Dozois, K. A. Kelly, and J. H. Pemberton, "J ileal pouch-anal anastomosis for chronic ulcerative colitis: complications and long-term outcome in 1310 patients," British Journal of Surgery, vol. 85, no. 6, pp. 800-803, 1998. 